\title{
Societal Self-observation in the Time of Datafication: Interfunctional Analysis of the Chilean Open Data Web Portal
}

\author{
Maximilian Heimstädt ${ }^{1}$
}

\begin{abstract}
Datafication, the technological development that emerged out of computerization and global interconnectedness, has spawned new forms of societal self-observations. In the present article I turn to the example of Open Data web portals - specialized websites that make large amounts of governmental datasets publicly available - to show how they relate to the status quo of social research on functional differentiation. For my analysis of the Chilean Portal de Datos Públicos I developed a method to link metadata categories from the web portal to a hard-core list of ten function systems. My results confirm literature, which finds economized or politicized forms of societal self-description. Moreover the results are in line with studies that show the vanishing role of religion. Interestingly, my study finds health to be of high importance - I might even speak of a "healthized" self-observation - which I argue is at odds with a negligible representation of the function system "sport" within the self-observation. For future interfunctional social research in the time of datafication, I recommend sharpening the empirical approach by exploring emerging text-asdata methods.

Keywords: Datafication, functional differentiation, interfunctional analysis, self-observation, social systems, Open Data
\end{abstract}

\section{Introduction}

We rely on self-observations from within society to learn about society, as there is no conceivable point outside of society from which to observe it (Luhmann, 1984). When self-observations overemphasize some elements described, whilst diminishing others, the ability to make a fair evaluation of the state of society is constrained. When selfobservations are the only way for second-order observers to learn about society, these self-observations may become self-fulfilling prophecies. An economized selfobservation can lead to the reproduction of an economized society, just because the observer struggles to imagine alternative forms of society (Roth, 2015). The more and especially the more resourceful second-order observers a self-observation has, the more likely it is to become self-fulfilling. Modern times have borne new forms of selfobservation. Many of these new and influential self-observations can be linked to farreaching developments in information technology. Firms, research labs and computerization movements have helped to diffuse IT innovation through almost every household and organization (Kling \& Iacono, 1990; Iacono \& Kling, 1996). An

1. Department of Management, Free University of Berlin, Boltzmannstraße 20, 14195 Berlin. Email: m.heimstaedt@fu-berlin.de 
inherent consequence of this diffusion is what Lycett (2013) calls the datafication of the world: Data is dematerialized and thereby separated from the physical world. Once dematerialized, data can be easily moved around and broken down, making it easier to recombine in any desired way. Being aware of the role of self-observations within society, I find it necessary to draw analytical attention to new forms of selfobservation in the time of datafication.

In this empirical study I direct my attention to the Chilean Open Data web portal Portal de Datos Públicos. ${ }^{2}$ Open Data is a public policy project that follows the ideas and assumptions underlying Free and Open Source Software. Proponents of Open Data argue that government datasets should be published in formats and under licenses that allow everyone to use and remix it. Whether this idea provides what has been promised in various discourses - specifically: transparency, collaboration and economic growth - might remain unanswered at this point. However, the concept has spread across nation states on all continents within the last five years. Central in this diffusion has been the establishment of national Open Data web portals. These portals are usually run by a government agency and provide access to datasets ranging from census and budget data to environmental and geospatial data. To evaluate the state of an Open Data portal or any other self-observation, I need a comparative momentum. I therefore draw on Luhmann's (1977) concept of a functional differentiated society, consisting of dissimilar but equal subsystems. To traverse Luhmann's ideas to an empirically fruitful analysis, I turn to a recently composed list of ten hard-core function systems prevalent in modern society (Roth \& Schütz, 2015). I therefore set out to answer the research question: What is the role of the different function systems within the Chilean Open Data web portal?

The study is structured as follows: I begin by showing the necessity and feasibility for societal self-observations to be analyzed through the lens of functional differentiation. Against this background I use Bowker and Star's (1999) work on categories to propose an empirical method for the analysis of Open Data portals as a form of modern self-observation. I then report my findings from applying the method to the Chilean Open Data web portal Portal de Datos Públicos. I conclude with a critical discussion of my findings and their implications for the development of interfunctional social analysis.

\section{Theoretical Initial Conditions}

\subsection{Analyzing Functional Differentiation}

As part of his theory of social systems, Niklas Luhmann described the functional differentiation of modern society as the latest evolutionary step of societal differentiation. For Luhmann, differentiation in a system is the "reduplication of the difference between system and environment within systems" (Luhmann, 1977, p. 31). Systems are thereby performing "the reproduction of the system in itself, multiplying

2. http://datos.gob.cl/ 
specialized versions of its own identity by splitting it into internal systems and environment" (Luhmann, 1977, p. 31). A broader historical contextualization shows an evolutionary development of societal differentiation. Roth and Schütz (2015) categorize different phases of this development along two dichotomies: equal/unequal and similar/dissimilar subsystems. The first known accounts of society are families and tribes. Across society, these subsystems were relatively similar and there were no institutionalized hierarchies or predominance of any of these families or clans. With the agricultural revolution, the subsystems of families and clans persisted but started to form settlements around strategically relevant loci (e.g., access to water or fertile ground). In addition to a differentiation in families and clans, now proximity or distance to these strategic locations created another differentiation of society in still similar yet unequal (center/periphery) subsystems. When the first hierarchical social orders formed (e.g., the Indian caste system), this process added another layer of differentiation - this time into hierarchically unequal and qualitatively dissimilar subsystems. "Functional differentiation," Luhmann (1977, p. 34) argues, "is the latest outcome of socio-cultural evolution." Today, subsystems of society cannot only be formed by segmentation, centralization, and stratification, but by the distinction of dissimilar, yet equal, function systems. Despite or just because of their importance to modern society, there is little consensus on where to find and how to name the function systems.

To move Luhmann's ideas from theoretical musings to the empirical workbench (and back) we need operationalizations. Roth and Schütz (2015) review and discuss different propositions which social systems qualify as a function systems and eventually present a canonical list of ten: 1 . the political system, 2 . the economy, 3 . science, 4. art, 5. religion, 6. the legal system, 7. the health system, 8. sport, 9. education, and 10. the mass media system. Based on their list, the authors claim that this pragmatic approach to functional differentiation is fruitful ground for an agenda of interfunctional comparative social research. Endeavors of this kind, they argue, could not only link Luhmann's theoretical work with more recent streams of social analysis, but also generally further our understanding of the interrelatedness of the societal building blocks.

\subsection{The Role of Self-observations in Interfunctional Analysis}

To analytically compare the function systems of modern society, I need a description of society on which to base my analysis. In the realm of Luhmann's theory of social systems however, a description of society is not possible from anywhere outside the system, as society incorporates all the social. I therefore need to observe an observation that happens within the system. Yet, in line with Fichte's metaphor of the eye that cannot see that it sees (Luhmann, 2002), this observation cannot be my own. What I need is an observation of a second order, an observation of a self-observation that happens within society.

Although self-observations eliminate the blind spot of first-order observations, they entail two limitations that we have to be aware of. First, self-observations are 
always simplified representations of the actual complexity of the system (Kittel, 1993). Alfred Korzybski (1933) popularly framed this phenomenon: "the map is not the territory." Self-observations of society are maps, created by an observer who had limited access to information. Although the observer did not include all possibly existing information in his observation, he might strive to develop a model that is adequate for use in certain contexts. Kittel (1993) moreover notes that many modern self-observations have not been designed in the halls and offices of academia but rather develop through day-to-day interactions on the street. They might therefore lack some of the systematic and reflective balancing that might be achieved by particularly trained and sensitized researchers. As Kittel (1993) explains, self-observations of society come with a second limitation: They cannot be compared with other candidate descriptions based on a higher-quality criterion. It is impossible to control for the validity and accuracy of these self-observations. I can summarize and must be aware in my analysis that self-observations present society with reduced complexity and that I have no criterion to evaluate how close they are to the object of observation. Selfobservations originating in one of the function systems can re-enter this or the other function systems of a higher-order system. The more a certain self-observation spreads throughout the system, the more other diverging self-observations move to the back.

As self-observations are always reductionist, deviations between the model and the object under observation are inevitable. These deviations create tensions, which in turn might trigger two types of adaptation processes. On the one hand the deviations between system and self-observation may lead to a partial reconfiguration of the selfobservation. This is the type of process that we find when natural scientists adapt their theory to improve the fit to their data. On the other hand and in the case of social systems, deviations may also work in the other direction and lead to modifications of the system instead of the self-observation. For example we can imagine a recursive dynamic through which over time the system may become more and more similar to the dominant self-observation, as Ferraro, Pfeffer and Sutton (2005) show in their study of self-fulfilling economic theories in management practice. As another example we can imagine the opposite, a self-defeating recursive dynamic in which the self-observation itself prevents the system from becoming alike. ${ }^{3}$

In the previous section, we showed that functional differentiation is a fruitful lens to use when examining modern society. A pragmatic approach to function systems helps us operationalize this lens. In this section, we have shown that we need to base an interfunctional analysis of society on self-observation. Epistemologically, we cannot determine at which points certain self-observation might deviate from the social system, as there is no external criterion for validation. However, what we can do is examine how the different function systems are represented within the self-

3. An interesting side note to the latter is given by Felin and Foss $(2009$, p. 664), who explain that "a brief and rough analysis of the top organizational journals ... reveals that the term self-fulfilling prophecy is invoked over $2000+$ times in these journals ... on the other hand, 'self-defeating' (or 'destroying' or 'negating') appears only 24 times." 
observation and compare the results with other self-observations to see at which points they differ or converge. In this study, I have therefore conducted an interfunctional analysis of a modern self-observation in the time of datafication to learn in what way it differs from other types of self-observation by answering the research question at hand: What is the role of the different function systems within the Chilean Open Data web portal? In the following chapter, I outline why the proclaimed time of datafication provides a worthwhile research context, why the Chilean Open Data web portals comprise a suitable case, and how I have operationalized the theoretical concepts at hand.

\section{Method}

\subsection{Research Context: The Time of Datafication}

Datafication is the technological development that emerged out of computerization and global interconnectedness. Since the introduction of the first personal computers in the 1960s and 1970s, firms, research labs, and computerization movements have helped to diffuse this technological innovation through society and into almost every household and organization (Kling \& Iacono, 1995; Iacono \& Kling, 1996). Whilst talk of a computer "revolution" flooded the mass media, science and technology scholars started to discuss "the relationship between the use of computer-based systems and transformations in parts of the social order" (Kling, 1991, p. 342). Through mainframe and subsequently personal computers, it was possible to generate and store more data more quickly than in previous un-computerized times. What I consider a second necessary causal condition for modern datafication is the global interconnectedness of data repositories. Several popular accounts describe how the development of the Internet (Hafner \& Lyon, 1998) as a technical layer for social interconnectedness (Castells, 1996) and further developments in data collection and processing (Boyd \& Crawford, 2012) cumulate in modern datafication of the social.

Lycett (2013) characterizes datafication based on two factors: First, modern data is dematerialized and thereby separated from the physical world. Second, once dematerialized, the data can be easily moved around and broken down, making it easier to recombine in any desired way. When I now assume that datafication has implications for our functionally differentiated society, I find confirmation for my hypothesis within the essentials of social systems theory itself. In his major contribution Die Gesellschaft der Gesellschaft, Niklas Luhmann (1997) already foreshadows the implications of computerization and the subsequent datafication of society. Luhmann brings the proliferation of computer communication in line with societally disruptive technologies like the art of writing or the printing press and argues that new forms of communication inevitably lead to new forms of selfobservation.

In a recent study, Roth (2014) picks up this notion and conducts an analysis using the tool Google Ngram Viewer. By tapping into the enormous database of Google Books, he conducts a keyword search to show the variations in dominance of certain 
function systems over time and across different cultures. With my study, I am contributing to this kind of interfunctional comparative analysis in the time of datafication, while also contrasting its wide, cross-cultural, and longitudinal approach by zooming in on a country-specific self-observation at a certain point in time. This single-case study approach (Yin, 2013) complements Roth's approach in two ways: Firstly, we obtain a more clear-cut picture of the observer who we are observing. When tapping into the Google Books database, we must refute the assumption that all function systems have contributed equally to this self-observation, and we need to assume that function systems with primary access to the production of books are overrepresented. However, as access might have shifted significantly over time, the picture of the observer in this kind of longitudinal analysis is blurred. By focusing on the single case of a government-run Open Data web portal at a certain point in time, my study allows for a deeper understanding of the dynamics that have led to the creation of a certain self-observation. Secondly, drawing on a single case and an amount of data can still be processed by a human researcher, I have been able to combine quantitative measures with qualitative methods. Whilst the text analysis by Roth (2014) was limited to querying the database for predefined keywords, my approach allows me to link parts of the self-observation to certain function systems by interpreting them using contextual knowledge and my personal sensitization in the research field. Before explaining this operationalization in depth, the following section provides an introduction to the case study at hand.

\subsection{Case Study: Open Data Web Portal}

The field of the interfunctional comparative analysis of social structure is still young, and I intend my study to add to the development of a more comprehensive research program, by demonstrating the potential of an in-depth single-case study analysis. As an example of a self-observation in the time of datafication, I analyze the national Open Data web portal of Chile: Portal de Datos Públicos. Open Data is a public policy concept closely linked to the principles of Free and Open Source Software. Through a variety of licenses it is assured that the Free and Open Source Software remains free and open, which means that everyone should be able to look into the source code and be able to change it. In the early 2000s this paradigm of digital openness outgrew the realm of software and was transferred to the realm of digital government datasets (Tkacz, 2014). From then on Open Data described not only the idea but also governmental efforts and projects to publish their digital datasets under licenses that allow everyone to use and remix them. The governmental narratives for legitimizing Open Data projects revolve around transparency, collaboration and economic growth. In recent years many national governments have launched an Open Data web portal. These portals are specialized websites that are run by a government agency and provide access to datasets ranging from census and budget data to environmental and geospatial data.

We consider the case of Chile's Open Data portal to be a particularly interesting one, as Chile had already been the stage of an advantageous self-observation around 
40 years ago. In 1971 on behalf of the Allende government, the British consultant Stafford Beer received the mandate to transfer his ideas of management cybernetics to the entire national economy. Central to this socialist-utopian endeavor was his plan to control the country's industrial production through a number of key indicators, which-through the use of telex-machines - should be sent from the largest factories in the country to be consolidated in a futuristic control room in Santiago de Chile. Decision makers inside the control room should have been able to oversee the indicators and instruct countermeasures where significant deviations occurred. Although Allende publicly framed the project with socialist political rhetoric, it was closely modeled after Beer's previous works on management cybernetics and his viable system model (Medina, 2006). Only two years later, however, the Allende government - and with it the so-called Project Cybersyn-came to a sudden end (Medina, 2011). What Beer created was not only a self-observation of Chilean society in the early 1970s, but also a non-digital predecessor of today's ubiquitous datafication.

This brief detour into the early days of management cybernetics enriches our study in two ways: Firstly, it opens up comparative momentum to our singly-case study on the Chilean Open Data web portal. In Allende's Chile, the government attempted to observe and describe the economic system by selectively collecting data about it. Now, more than 40 years later, the government is again setting out to create a model of its environment based on the data it is collecting. This time, however, the collection is not limited merely to the economic system, but is open to all function systems. Secondly, the detailed story of how Beer designed Project Cybersyn informs the operationalization of our research interest. Although he intended to create a holistic model, Beer's team started by connecting only 30 nationalized industries to the telex system at first and was able to raise this number to over 100 within two years, yet while still representing only $26.7 \%$ of all nationalized enterprises. Moreover, to reduce the complexity of the system, Beer's team selected just five variables, which they regarded as critical to describing production (Medina, 2006, p. 588). Within Project Cybersyn, the selection of connected sites and the small selection of variables reduced the complexity and determined the model at hand. In the case of Open Data, web portals therefore have to pay attention to which data providers (think national enterprises) are represented in the portal, and which types of data (think production variables) they contribute.

\subsection{Operationalization}

With this study I want to examine what role the different function systems play within the Chilean Open Data web portal. I therefore need to operationalize the idea of interfunctional comparative analysis of social structure to apply it to the Portal de Datos Públicos. This operationalization needs to take place within the framework of second-order observations: In this study I observe how the political system (in the gestalt of the Chilean government) observes the entirety of function systems in Chile. Just as reading a book is a form of second-order observation, I engage with-I 
read - the Chilean Open Data web portal. The rationale for my second-order observation is that the Open Data web portal (as a first-order observation) serves as an irritation to the other function systems and influences their development. An intended or unintended maladjustment of the Open Data web portal might therefore lead to dynamics of (e.g., self-fulfilling or self-defeating) alteration within all of the subsystems.

The central problem of the operationalization is how to link empirical data to the canonical function systems. Only through this link can I determine which of the function systems are more or less important in the self-observation. In his study based on the corpus of Google Books, Roth (2014) has used the frequency of words associated with the ten function systems as a measure of their relative importance. However, as an Open Data web portal is comprised of qualitatively different data than Google Books, this process has to be rethought. The Chilean Open Data web portal holds datasets in various formats, sizes and degrees of completeness. Most of the datasets are in the form of spreadsheets, yet they lack any kind of standardization. To bridge the gap between the noisy and non-standard forms of these datasets and the list of societal function systems, I decided to base my analysis on the metadata available for each of the datasets. The metadata thereby serves as a proxy that ensures a minimum of standardization but still leaves room for interpretation by the researcher. The use of metadata significantly narrows the gap between datasets and function systems. However, we still need a concept that ultimately links them up.

As a remedy for this, I need to turn to Bowker and Star's (1999) work on classification schemes. They explain that

a classification is a spatial, temporal, or spatio-temporal segmentation of the world. A 'classification system' is a set of boxes (metaphorical or literal) into which things can be put to then do some kind of work-bureaucratic or knowledge production. (Bowker \& Star, 1999, p. 10; emphasis in original).

Following their idea, I set out to sort the metadata of the individual datasets in the Open Data web portal into ten boxes, each labeled with the name of a function system. However, not all of the metadata contained information suitable for interpretation and sorting - I first had to separate the signal from the noise. In a manner analogous to Stafford Beer's team 40 years ago, I tried to reduce the variables at hand and to identify a small subset of variables that would provide me with the information I was looking for. Figure 1 shows the four steps through which I compressed the 18 available metadata categories into a final subset of three. 


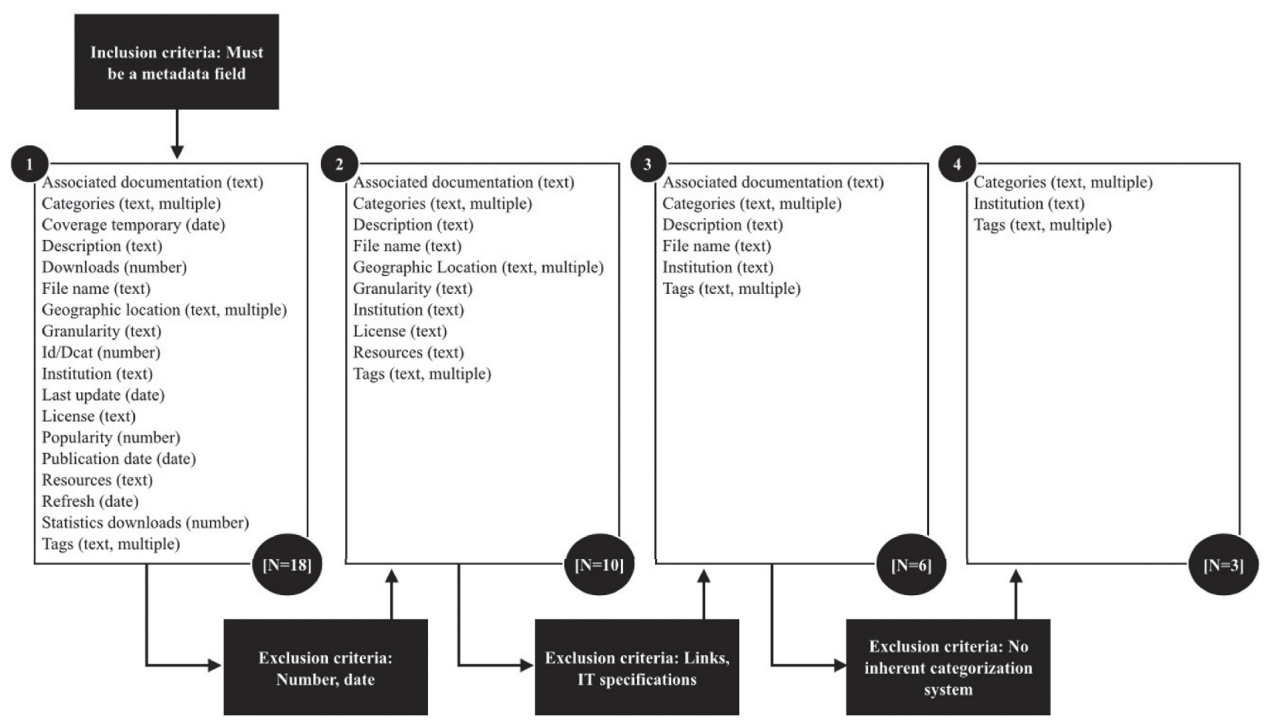

Figure 1: Metadata selection process

In the first step of the process, I assembled a list of all metadata fields and ended up with 18 entries. For each of these fields I then added whether it carries a humanreadable word (text), a date or a year (date), a number, or a mixture of numbers and letters (number) to quickly identify whether I would be able to interpret its content in relation to the function systems. When the metadata field could be filled with multiple distinct strings, I added multiple to the description to indicate that metadata in this field could relate to more than one function system. In the second step, I excluded all the fields that carried numbers or dates from the list. The numbers were basically identifiers necessary for database entries but with no information about the provenance of the data. The dates mainly related to the upload date of the dataset. However, in this analysis, I am interested in the status quo of datasets on the web portal and not in the process of their uploads over time. This step reduced the number of entries on the list to ten. In the third step, I excluded all the fields that represent links to other datasets or technical specifications. Information in these fields again helps to understand the form of the data, but I am not aware of any connection between the form and certain function systems. This resulted in a list of six textual metadata fields. In the fourth step, I excluded all fields that do not carry an inherent categorization system. For example, the field File Name carries textual information, but every file name is different. There is no inherent classification system. However, the field Institution can only be filled with a limited number of options and one institution can be the source of many different datasets. The methodological considerations of this last step regarding the potential use of text-as-data methods (Grimmer \& Stewart, 2013) are discussed in the final section of this essay. This 
process of reducing complexity results in three metadata fields that are open to human interpretation. Through interpretation, their content can be linked to the function systems. Chile's Open Data web portal is a self-observation that consists of datasets. Linking these datasets to the function systems has therefore enabled us to understand the self-observation as a composition of different function systems, in which some function systems are more while others are less prevalent. In the following section, I describe my interpretative process and present my findings to illustrate - and thereby address the research question of this study - the role of the function systems within Chile's Open Data web portal.

\section{Findings}

The Chilean Portal de Datos Públicos promises its visitors that they "will find sets of public government information" and that "the aim of this tool is that people have access to information based on which the government makes decisions for public policy ..." (see http://datos.gob.cl/). From these brief statements we learn that through the portal the Chilean political system describes other function systems of Chilean society, including itself. Portal de Datos Públicos was launched in mid-2011 and started with just a handful of datasets. By the end of May 2012, 51 datasets were available, and by the time of data collection (July 6, 2015) 1,210 datasets were uploaded.

Table 1: Categories and Function Systems

\begin{tabular}{ll}
\hline & Category \\
\hline Value & Function System \\
\hline Business & economy \\
Communications & mass media \\
Culture & art \\
Education & education \\
Emergencies & political system \\
Employment & economy \\
Finance & economy \\
Government & political system \\
Health & health system \\
Industry & economy \\
Policy & political system \\
Recreation & health system \\
Security & health system \\
Science & science \\
Technology & science \\
Tourism & health system \\
\hline
\end{tabular}


Table 2: Tags and Function Systems

\begin{tabular}{ll}
\hline & Tag \\
\hline Value & Function System \\
\hline Budget & political system \\
Budget Execution & political system \\
Education & education \\
Employment & economy \\
Expenses & political system \\
Government & political system \\
Governorates & political system \\
Health & health system \\
Higher Education & education \\
Hospitals & health system \\
Law & legal system \\
Mining & economy \\
Work & economy \\
\hline
\end{tabular}

Table 3: Institutions and Function Systems (Excluding Political System)

\begin{tabular}{ll}
\hline \multicolumn{2}{c}{ Institution } \\
\hline Value & Function System \\
\hline Ministry of Education & education \\
Ministry of Finance & economy \\
Ministry of Health & health system \\
Ministry of Justice & legal system \\
Ministry of Labour and Social Welfare & economy \\
Ministry of National Heritage & art \\
Ministry of Transport and & mass media \\
Telecommunications & \\
National Council for Culture and the Art & art \\
\hline
\end{tabular}

I analyzed these datasets based on the three metadata categories derived via the process of operationalization. Each of the metadata categories has different content for each dataset. At this point, it might help to think of the content as values, although it is textual and not alphanumeric. Therefore, each metadata field can have different values, and these values, in turn, can be connected to the function system. After matching all the values for the three metadata categories with function systems, we can then take a step back and examine the distribution. Tables 1-3 show how I linked these values to the function systems. In each of the tables, the value in the left column 
is matched with a function system in the right column. Similarly to Stafford Beer's team, I needed to balance complexity reduction against the danger of oversimplification. As Beer's team decided to use five production indicators per industrial plant (instead of all of them or just one), my reading of the self-observation is based on the three metadata categories. It follows that, in the remainder of this section, I briefly present the results for each of these categories to illustrate my method of analysis before I move on to the discussion of the aggregated results in the following section.

The first metadata field is named Category and sorts the datasets into what I might call a domain of life. These categories, or domains, range from Government and Education to Society or Community. In total there are 23 of such domains or values. In some cases the connection between the domain and an associated function system seems rather obvious. One example of this is linking the category Government to the political system. However, many categories were difficult to relate to a single function system. In these cases I had a look at a convenient sample of datasets within the categories. When I found that some of the datasets clearly relate to one of the function systems, I established the link. An example of this is when I linked the category Communication to the mass media system, as the category contains datasets like a list of radio broadcasting concessions granted or a list with the location of mobile phone masts. In total I was able to link 16 out of 23 values to function systems (see Table 1).

The second metadata field is named Tag and associates the individual dataset with keywords - otherwise known as tags. In total there are 822 tags associated with 1,210 datasets, in which the majority of tags is only associated with a single dataset. To make sense of and to be able to interpret this large number of tags, I decided to only include in my analysis tags that are associated with ten or more datasets. I think that this threshold qualifies the remaining tags as an inherent classification scheme, which I used in my selection process for metadata fields. As with the categories, I again interpreted the tags, in some cases looking at the datasets associated with them, and finally linked each of them to the appropriate function system. Some tags could be linked with little doubt, for example Health (health system), Higher Education (education) or Law (legal system). Other connections, like linking the tag Budget Execution to the political system were less obvious but plausible ex post. Out of 30 tags I was able to associate 13 with a function system (see Table 2).

The third metadata field is named Institution and names the formal organization in which the dataset has been created. In contrast with the other two metadata fields, this one describes formal organizations, which generally cannot be associated with a single function system alone. Besides one exception (Public Enterprise), all institutions in this metadata field are organizations of the Chilean government (e.g., Ministry of Education). Therefore all of them can be linked to the political system; moreover, some of them can also be linked to another function system (e.g., education). For this reason I excluded the political system from my scheme and looked at which of the institutions can be linked to another function system. Out of 25 institutions I was able to link eight to function systems other than the political system. 
For the Ministries of Education (education), Finance (economy), Health (health system) and Justice (legal system), the link to a function system was directly visible. It also appeared inevitable to link the National Council for Culture and the Arts to the art system. When thinking about the Ministry of National Heritage, I considered its role in education but came to the conclusion that it operates mainly with the aesthetic codes of innovation and imitation (Roth \& Schütz, 2015) and therefore has to be linked to art as well. Out of 25 institutions I was able to link eight to function systems other than the political system (see Table 3).

In this section, I applied the operationalization of interfunctional analysis to Chile's Open Data web portal. My aim was to show how the portal, as an example of self-observation in the time of datafication, can be read as a composition of function systems. Tables 1-3 show the distribution of function systems for three different metadata categories. Although these three categories have been selected as those carrying the most information for our purposes, I could not match every value in each category with a function system due to limitations in the data. In the following section, I have therefore merged the results from these three metadata categories into a weighted list of the ten hard-core function systems. This has not only helped me to holistically interpret the Open Data web portal as a self-observation, but, moreover, it has made my results comparable to the relevant literature.

\section{Discussion}

The purpose of interfunctional analysis is to describe and compare the existence and weighting of different function systems within the self-observations of society. In his study on different forms of capitalism, Roth (2015) ranked the individual function systems to visualize their over- and under-emphasis within different types of society. In the sections above, I have described the measures I took to condense the abundant information on the Open Data web portal into categories and variables that allowed me to interpret it as a self-observation in the time of datafication. Due to the compromises I had to make (using only three out of 18 metadata categories, not linking all of the values per category with a function system), I have refrained from the idea of bringing the function systems into a discrete order. The reason for this is that I do not think that I have sufficient data points to make the fine-grained evaluations needed to pick one out of the 3,628,800 options for how to rank the ten function systems (Roth, 2015).

As an alternative way to present and interpret my results, I decided to group the function systems into three groups of high, medium, and low importance in the selfobservation. The grouping is based on the number of links established between the function systems and the metadata. The group with function systems of high importance consists of the political system, the economy and the health system. Of medium importance in the Portal de Datos Públicos are education, the legal system, science, art and mass media. I found no topic or tag that could be associated with sport or religion, and consequently their importance is considered to be relatively low. Roth 
(2015) uses the metaphor of the studio sound board to show the interaction between function systems in a society. Figure 2 shows an adapted and modified version of the controller that Roth proposed for displaying the relative importance of function systems in a self-observation. The equalizer visualizes the role of the different function systems within the Chilean Open Data web portal. I modified the equalizer in two respects: Firstly, I reduced the options per function system knob from ten to three to represent high, medium, and low importance. Secondly, I visually separated the political system from the other nine function systems. By doing this, I was able to account for its position in the group of highly important function systems while, at the same time, accounting for its predominant position in this self-observation, which was produced from within the political system itself. Through the Chilean Open Data web portal the Chilean political system performs reflexive observation, and at the same time observes Chile's other function systems. I could therefore assume - and this was also confirmed by the data - that the political system is highly overrepresented in this self-observation. This becomes obvious when looking at the metadata category Institution, in which every entry has a link to the political system (see Table 3). By isolating the political system visually I can acknowledge its high importance in this self-observation but at the same time clear my view for imbalances amongst the remaining nine function systems.

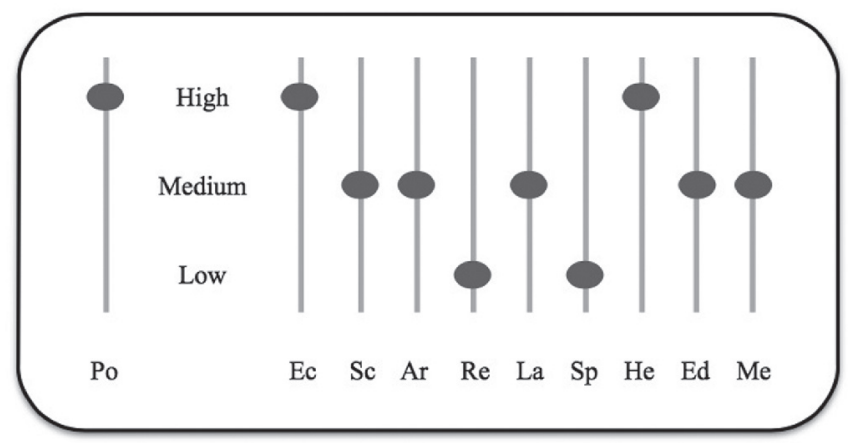

Figure 2: The Function System Equalizer Adjusted to the Chilean Open Data Web Portal (adapted and modified from Roth, 2015)

The equalizer in Figure 2 shows how the different function systems are mixed together in the Chilean Open Data web portal as a modern self-observation. To determine the impact and relevance of this particular self-description, we now need to pose the question of how this result resonates with our understanding of function systems in general and the function system mix of other self-descriptions. Kittel (1993) claims that in a functionally differentiated society, theoretically all function systems are equal (in contrast to the unequal subsystems of a stratified society). From my point of view this equality holds true in one way but not another. There is equality between the function systems in that they all possess what Luhmann calls operational closeness: The only way they can change their structure is through their own 
operations (1997). However, as many authors have described, this idea of equality does not necessarily hold true when it comes to their contribution to the overall society. It has been found in various studies that some function systems dominate and thereby silence others (Willke, 1989).

The same is reflected in the Chilean Open Data web portal: In the group of function systems with a high importance in the self-observation, I find the political system, the economy and the health system. The top ranking of the economy does not come as a surprise and resonates with the long-standing claim of an economized society (Polanyi, 1957). When taking into consideration the prominent position of the political function system, I end up with what Roth $(2015$, p. 109) describes as an "economy-biased political system." More surprising than seeing the economy and the political system ranked high is the prominent role of the health system. When Roth (2014, p. 34) lists "prominent modern trend statements and predictions," he names "secularization, politicization, economization, and mediatization," yet there is no reference to a potentially healthized society. Williams (2002, p. 185) however discusses this phenomenon and tries to differentiate between societal "medicalization and healthization: the former advancing biomedical causes and interventions, the latter advancing lifestyle causes and behavioral interventions." The prominent role of the health system within my analysis, together with the dissimilar yet not mutually exclusive definitions of medicalization and healthization, calls for further research in this domain that is potentially connected to the notion of biopolitics and the modern logic of self-responsibility (Foucault, Ewald, \& Fontana, 2010).

In the group of function systems of medium importance within the selfobservation, I find education, the legal system, science, art and the mass media. There have been individual studies that claim the overemphasis of some of these systems within society, however, I do not want to make any claims concerning them based on the empirical data from my single case study. Reaching the group with the lowest level of importance among the function systems, I find that both sport and religion have not even been associated once with any category of Portal de Datos Públicos. Although religion is nonexistent in this self-observation, its status as a function system is without question. This axiomatic statement becomes obvious when looking at the prominent role of religion in occidental medieval or different contemporary societies that are considered theocracies (Roth, 2014). An explanation for the negligible role of religion in the Open Data web portal might be found in the role of secularization in modern democracies. As Open Data web portals are an outcome of the political system, it can be seen as an indicator of social equality that religion does not play a role in how this system observes society. I want to close this discussion with the curious case of sport as a function system. Roth and Schütz (2015) frame sport as a function system whose status is principally uncontested, and yet there remains discussion about the naming of its code and function. The authors decide that the function system communicates through the code success/failure and has mobilization (Roth \& Schutz, 2015) as its main function. Recent years have seen ever-growing international sports events like the FIFA World Cup or the Olympic games arranged or 
at least widely supported by the political system. In this light it seems counterintuitive that the function system plays a negligible role in the Open Data web portal. I think there could be two solutions for this divergence between expectations and findings. A pragmatic explanation could be that the Institution responsible for sport-related datasets has for some reason failed to upload data to the portal even though the data plays a role in the political system. When following this explanation further we would have to differentiate between the datasets that every institutions was supposed to upload and actually uploaded. The second more fundamental explanation could be that I need to re-discuss the differentiation between the health system and sport. As I have pointed out above, it seems unclear whether the health system incorporates health as a result of medical treatment, health as a result of lifestyle, or both. When I look at sport apart from elitist events like the Olympic games or the World Cup, I might think that in many cases sport communication does not happen through the code of success/failure but ill/healthy or rather unhealthy/healthy. When looking at the functions of the respective systems, I find that mobilization (sport) and restoration (health system) might overlap, for example, when white-collar workers visit the gym in the evening to improve their running skills but at the same time want to cure their backache. This problematic overlap between function systems might be an explanation for the very prominent position of health as well as for the underrepresentation of sport.

In conclusion, the aggregation of our tripartite analysis and its visualization in the form of the function system equalizer has helped us to understand the mix of function systems represented in the Chilean Open Data web portal. Although most of the particularly high or low controller positions resonated with the existing literature, I found other positions that were rather surprising. Methodological limitations that could have impacted these results as well as inspiration for further research, will be briefly touched upon in the final section.

\section{Limitations and Further Research}

In this study I have examined the Chilean Open Data portal as modern selfobservation through the lens of functional differentiation. I find that this selfobservation has a strong bias toward the political and economic function systems, as expected from the literature. However, I also find that the health system as a function system occurs very prominently. Furthermore, I find that religion and sport — although part of Roth and Schütz's (2015) list of hard-core function systems-play no significant role within the self-observation. The main limitation of the study simultaneously serves as a call for further research: In the field of interfunctional studies of society, I see great potential for text-as-data methods (Grimmer \& Stewart, 2013), which in my case might be able to process all metadata of the Open Data web portal and match it with predefined keywords and categories fitted to the different function systems. In my study I excluded all textual metadata that did not carry a categorization system in itself (e.g., types of institutions) from the analysis. However, 
I think these fields might carry valuable information for adequately linking the data with the function systems. Through text-as-data methods, these textual descriptions can be transformed into chunks, which could automatically be matched with a list of keywords associated with the different function systems.

\section{References}

Bowker, G., \& Star, S. L. (1999). Sorting things out: Classification and Its Consequences. Boston: The MIT Press.

Boyd, D., \& Crawford, K. (2012). Critical questions for big data: Provocations for a cultural, technological, and scholarly phenomenon. Information, Communication \& Society, 15(5), 662-679.

Castells, M. (1996). The Information Age: Economy, Society and Culture: Vol 1. The Rise of the Network Society. Oxford, UK: Blackwell.

Felin, T., \& Foss, N. J. (2009). Social reality, the boundaries of self-fulfilling prophecy, and economics. Organization Science, 20(3), 654-668.

Ferraro, F., Pfeffer, J., \& Sutton, R. I. (2005). Economics language and assumptions: How theories can become selffulfilling. Academy of Management Review, 30(1), 8-24.

Foucault, M., Ewald, F., \& Fontana, A. (2010). The Birth of Biopolitics: Lectures at the Collège de France, 19781979. M. Senellart (Ed.). New York: Palgrave Macmillan.

Grimmer, J., \& Stewart, B. M. (2013). Text as data: The promise and pitfalls of automatic content analysis methods for political texts. Political Analysis, 21(3), 267-297.

Hafner, K., \& Lyon, M. (1998). Where wizards stay up late: The origins of the Internet. New York: Simon \& Schuster.

Iacono, S., \& Kling, R. (1996). Computerization movements and tales of technological utopianism. In R. Kling, (Ed), Computerization and Controversy (pp. 85-105). San Diego, CA: Academic Press.

Kittel, B. (1993). Die Selbstbeschreibung der gesellschaft: der begriff der nation als missing link der systemtheorie? Political Science Series \#4. Vienna: Institut für Höhere Studien (Institute for Advanced Studies). Paper retrieved November 3, 2015 from https://www.ihs.ac.at/library/publications/?tx_ihspeople_publications[publication]= 1440\&tx_ihspeople_publications[action] $=$ show\&tx_ihspeople_publications $\overline{\text { [controller] }}=$ IhsPublications\&cHash $=$ caa31de1a590d1181f704dc8bf8b6949

Kling, R. (1991). Computerization and social transformations. Science, Technology \& Human Values, 16(3), 342-367.

Kling, R., \& Iacono, S. (1990). Computerization movements and the mobilization of support for computing. In J. Berleur, A. Clement, R. Sizer, \& D Whitehouse, (Eds.), The Information Society: Evolving Landscapes (pp. 6283). New York: Springer.

Korzybski, A. (1933). A non-Aristotelian system and its necessity for rigour in mathematics and physics. In A. Korzybski (Ed.), Science and sanity: An introduction to non-aristotelian systems and general semantics (pp. 747761). Lakeville, CT: International Non-Aristotelian Library.

Luhmann, N. (1977). Differentiation of society. Canadian Journal of Sociology, 2(1), 29-53.

Lycett, M. (2013). Datafication: Making sense of (big) data in a complex world. European Journal of Information Systems, 22(4), 381-386.

Luhmann, N. (1984). Soziale systeme. Frankfurt, Germany: Suhrkamp.

Luhmann, N., \& Baecker, D. (2002). Einführung in die Systemtheorie. Heidelberg, Germany: Carl-Auer-SystemeVerlag.

Medina, E. (2006). Designing freedom, regulating a nation: Socialist cybernetics in Allende's Chile. Journal of Latin American Studies, 38(03), 571-606.

Medina, E. (2011). Cybernetic revolutionaries: Technology and politics in Allende's Chile. Boston: The MIT Press.

Open Knowledge (n.d.). Open knowledge definition. Website content retrieved July 6, 2015 from http:// www.opendefinition.org/

Polanyi, K. (1957). The great transformation. Boston: Beacon Press.

Roth, S. (2014). Fashionable functions: A Google ngram view of trends in functional differentiation (1800-2000). International Journal of Technology and Human Interaction, 10(3), 88-102.

Roth, S. (2015). Free economy! On 3628800 alternatives of and to capitalism. Journal of Interdisciplinary Economics, 27(2), 107-128.

Roth, S., \& Schütz, A. (2015). Ten systems: Toward a canon of function systems. Cybernetics and Human Knowing, 22(4), 11-31.

Tkacz, N. (2012). From open source to open government: A critique of open politics. Ephemera: Theory and politics in organization, 12(4), 386-405.

Williams, S. J. (2002). Sleep and health: Sociological reflections on the dormant society. Health, 6(2), 173-200.

Willke, H. (1989). Systemtheorie entwickelter Gesellschaften: Dynamik und Riskanz moderner gesellschaftlicher Selbstorganisation. Weinheim, Germany: Juventa-Verlag.

Yin, R. K. (2013). Case study research: Design and methods. Thousand Oaks, CA: Sage Publications. 
